\title{
LA NORMA ISO 9000 Y EL SISTEMA DE GESTIÓN DE LA CALIDAD
}

\author{
ISO 9000 AND THE SYSTEM FOR QUALITY MANAGEMENT \\ Adrián Alejandro Flores KonjA* \\ Docente Principal de la Facultad de Ciencias Contables \\ Manuel Alberto Hidalgo Tupia** \\ Docente Auxiliar de la Facultad de Ciencias Contables \\ Universidad Nacional Mayor de San Marcos-UNMSM / Lima-Perú \\ [Recepción: Marzo de 2014/ Conformidad: Mayo 2014]
}

\section{RESUMEN}

En el presente artículo, fruto de una investigación exploratoria que es parte de una investigación en marcha auspiciada por Vicerrectorado de Investigación de la UNMSM y con el apoyo del Instituto de Investigación de Ciencias Financieras y Contables de la Facultad de Ciencias Contables, se propone a los elementos que se deberían considerar en un Sistema de Gestión de la Calidad, apropiada a una Unidad de Posgrado, que es un requisito en el marco de la acreditación de la calidad de la educación, según el modelo de calidad de CONEAU.

El Sistema de Gestión de la Calidad como tal, contiene una organización coherente con los diferentes sistemas formulados en el marco del modelo de calidad de CONEAU, sistemas interconectados referidos a las 3 dimensiones (gestión, formación profesional y servicios de apoyo a la formación profesional), en los cuales los sistemas centrales son los de la planificación curricular.

Se analiza dicho sistema para la acreditación de la Unidad de Posgrado en concordancia con la norma ISO 9000, debido a su coherencia con el modelo de calidad de CONEAU, y propone las políticas generales de calidad consideradas apropiadas para la Unidad de Posgrado en la Facultad de Ciencias Contables.

\section{Palabras Clave:}

Sistema de gestión de la calidad; Unidad de Posgrado; acreditación; planificación curricular; enseñanza aprendizaje; modelo de calidad de CONEAU; norma ISO 9000.

\begin{abstract}
In the present article, the result of exploratory research is part of an ongoing investigation, sponsored by Vice President for Research of UNMSM and with the support of the Research Institute of Accounting and Financial Sciencesat the Faculty of Accounting, proposed that the elements should be considered in a System of quality Management, appropriate to a Postgraduate Unit, which is a requirement under the accreditation of the quality of education, according to the quality model of CONEAU.

System Quality Management as such, contains a coherent organization with different systems formulated under the Quality Model of CONEAU interconnected systems related to the 3 dimensions (management, training and support services to professional training), in which the core systems of curriculum planning. This system for the accreditation of the Postgraduate Unit in accordance with the ISO 9000 standard is analyzed because of their consistency with Quality Model of CONEAU and proposes general quality policies deemed appropriate for Unit Graduate School of Accounting.
\end{abstract}

\section{Keywords:}

System of quality management; Postgraduate Unit; accreditation; curricular planning; teaching - learning; Quality Model of CONEAU; Standard ISO 9000.

\footnotetext{
* Doctor en Ciencias Contables y Empresariales. Contador Público Colegiado - UNMSM. Email: afloreskonja@yahoo.es.

** Magister en Educación. Economista. Email: hidalgoman@gmail.com
} 


\section{INTRODUCCIÓN}

El Consejo de Evaluación, Acreditación y Certificación de la Calidad de la Educación Superior Universitaria - CONEAU, publicó en el diario oficial "El Peruano" de fecha 23 de diciembre de 2010, el Modelo de Calidad para la acreditación de Programas de Posgrado, modalidad presencial, y estándares para Maestrías y Doctorados, documento normativo que servirá de referencia para la presente investigación exploratoria, que forma parte del Estudio de Investigación: "Elementos del Sistema de Gestión de la Calidad para la Unidad de Posgrado de la Facultad de Ciencias Contables en marco del modelo de Calidad de CONEAU” (2014), auspiciado por el Vicerrectorado de Investigacion, cuyos integrantes son los autores del presente artículo, como parte de un equipo más amplio de investigadores.

En el artículo titulado "Modelos de Calidad en el marco normativo de la acreditación de las universidades del Perú" (Flores, e Hidalgo, 2012) se obtuvieron, entre otras, la conclusión que los criterios del modelo de calidad de CONEAU se basan en las nuevas tendencias de los enfoques administrativos, pues estos “... ha enfatizado en el concepto de la calidad; dentro de los enfoques de calidad total, la mejora continua, Balanced Scorecard, planificación estratégica y prospectiva, y ha llegado a la concepción que se requiere, no solamente el control de la calidad que nos permita verificar Ex - post si hubo o no calidad, sino anticiparnos para crear sistemas de gestión de la calidad".

Sin embargo, se ha constatado que no existen pautas metodológicas para la formulación eimplantación de sistemas de gestión de calidad a nivel de programas de posgrado. Dado el caso, que a la fecha, ninguna Unidad o Escuela de Posgrado han sido acreditadas por el CONEAU. En adición, CONEAU no propone un modelo específico de sistema de gestión de calidad de los muchos existentes, por lo cual la presente investigación tiene como tarea llenar un vacío en el conocimiento, de utilidad para la comunidad universitaria, científica y nacional.

Esta investigación exploratoria constituye un "Estado del arte" parcial sobre las variables de dicha investigación, en especial "Modelos de Gestión de la Calidad" en que se sustentan los Sistemas de Gestión de la Calidad. Se revisará los aportes conceptuales respectivos, su pertinencia y la coherencia entre los distintos modelos y el modelo de Calidad de CONEAU, que es el que se aplicará como base comparativa.

\section{MODELOS DE GESTIÓN DE LA CALIDAD UNIVERSITARIA}

Farro (2012: 186) plantea en el acápite de "Modelos de Gestión y Evaluación en la Educación $\mathrm{Su}$ perior Siglo XXI", cinco modelos, que se relacionan todos con el objetivo de la gestión, que es la calidad. Los modelos citados son:

- Modelo del Balanced Scorecard o Cuadro de Mando Integral, propuesto originalmente por Norton y Kaplan (2000).

- El Modelo de Excelencia EFQM, de European Foundation of Quality Management, (EFQM) o Fundación Europea de Gestión de la Calidad. (2010).

- Modelo de las Normas ISO 9000 y relacionadas, del International Standard Organization.

- Modelo de CONEACES, del Perú, aplicable a instituciones de educación superior no universitarias. (2009).

- Modelo de CONEAU, del Perú, Consejo de Evaluación, Acreditación y Certificación de la Calidad de la Educación Superior Universitaria (2008), que, como se ha señalado (Flores e Hidalgo, 2012), se relaciona con las tendencias administrativas modernas, que seguidamente será tratado.

Se debe puntualizar, que es este último modelo la base de referencia, no tanto por su mayor valor conceptual, sino porque es la base normativa aplicable a la Unidad de Posgrado de la Facultad de Ciencias Contables de la Universidad Nacional Mayor de San Marcos. Analizaremos, específicamente, el modelo de las Normas ISO 9000, a fin de determinar su pertinencia al caso presente (Facultad de Ciencias Contables) y su coherencia con el Modelo de Calidad de CONEAU, tratando de extraer de dicho análisis indicaciones prácticas para la aplicación de este sistema. 


\section{MODELO DE CALIDAD DE CONEAU Y SIS- TEMA DE GESTIÓN DE LA CALIDAD}

CONEAU plantea lo que llama explícitamente "Modelo de Calidad", proponiendo una definición de la calidad educativa de la UNESCO y estableciendo las dimensiones, factores, criterios y estándares del modelo de calidad, los que deberían ser tomados en cuenta en un sistema de gestión de la calidad.

CONEAU propone 3 dimensiones fundamentales:

1. Gestión de la carrera;

2. Proceso de formación profesional; y

3. Servicios de apoyo a la formación profesional.

Los factores y criterios de estas dimensiones están relacionados, de tal manera que los estándares comparten fuentes de verificación, pues algunos instrumentos son útiles para más de una dimensión, factor o criterio.

CONEAU (2010) incorpora explícitamente el "enfoque de procesos" de Deming, e implícitamente el enfoque sistémico, al igual que la Norma ISO 9000. El modelo de calidad para la acreditación de
Programas de Posgrado en la modalidad presencial. (CONEAU 2010,3):

“....aplica los principios de sistemas y enfoque de procesos (Figura 1). Este marco estructural promueve el orden, la sistematización, la evaluación objetiva y la autorregulación de la unidad académica de posgrado al facilitar la interacción de los procesos seleccionados que tienen lugar en ella y que le permiten alinearse al cumplimiento de los compromisos adquiridos con la sociedad en cuanto al conocimiento creado y los investigadores formados, expresados en los proyectos de investigación desarrollados y en la cantidad de egresados". Añade (loc. Cit.):

"A través del enfoque de procesos, los objetivos planteados pueden alcanzarse más fácilmente ya que los recursos y las actividades relacionadas están gestionadas como procesos, los cuales han sido relacionados bajo el principio de la mejora continua, aplicando el ciclo de Deming: planificar, hacer, verificar y actuar; enfoque que se potencia al considerar al proyecto como la unidad básica para una evaluación objetiva."

\section{Gráfico $\mathrm{N}^{\circ} 1$}

\section{Proceso de formación del estudiante de posgrado}

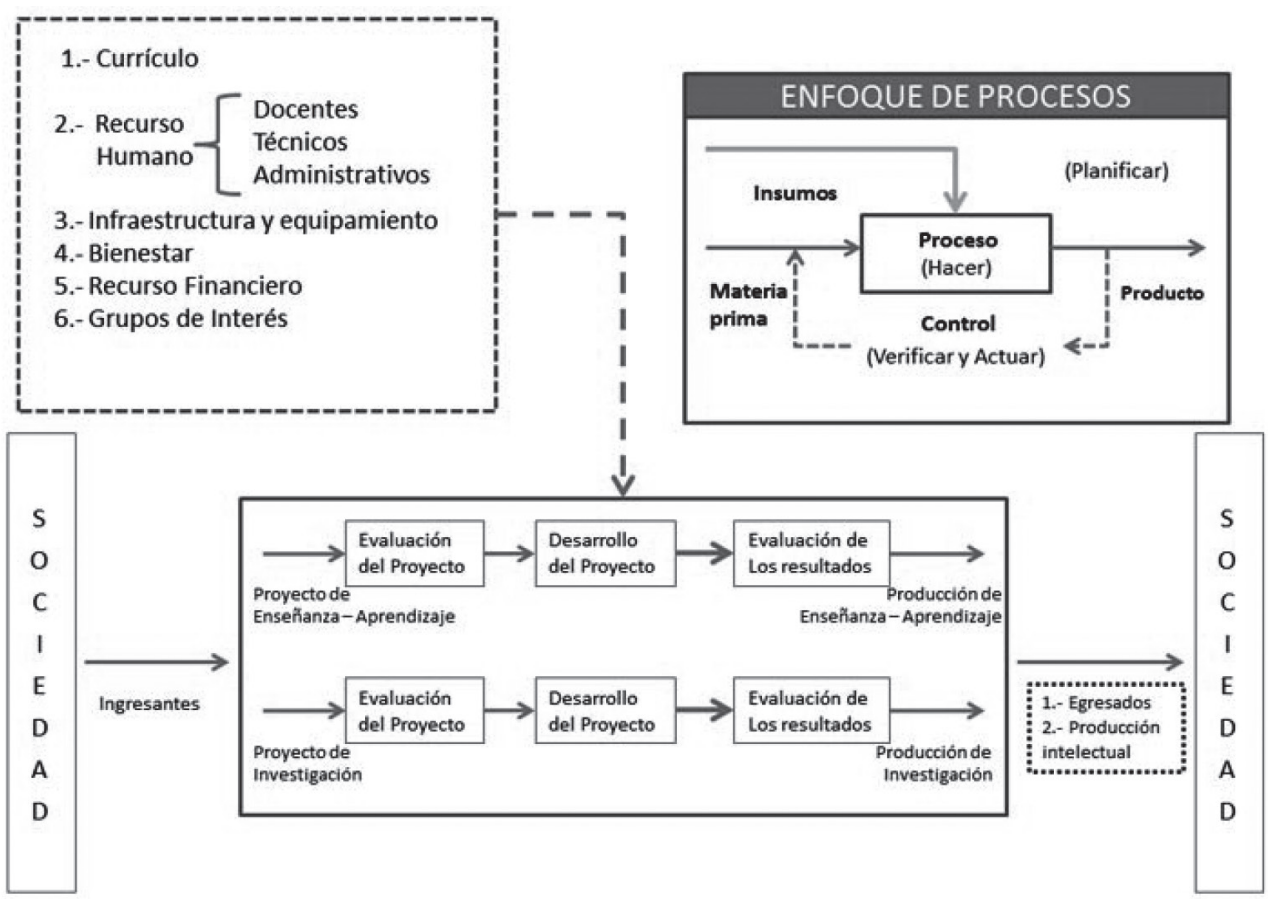

Elaboración: DEAC- CONEAU, 2010. 
CONEAU y su modelo propone una serie de "sistemas" por ejemplo: sistema de gestión de la calidad, sistema de seguimiento del egresado, sistema de evaluación del aprendizaje, sistema de información y comunicación, etc. Específicamente, el Sistema de Gestión de la Calidad como lo requiere expresamente en el Estándar $\mathrm{N}^{\circ} 8$, del Criterio 1 Organización, Dirección y Control, del Factor 1 (Planificación, organización, dirección y control) de la primera dimensión, Gestión de la Carrera. Dicho estándar señala explícitamente: "La Unidad Académica tiene un Sistema de Gestión de la Calidad implementado”.

El proceso principal es el de la Enseñanza-Aprendizaje, lo cual es igualmente priorizada en el modelo de CONEAU el que lo considera el proceso básico, en el que (Dimensión 2: formación profesional) concreta el mayor número de criterios (7 de 16), y que incluye investigaciones y extensión Universitaria y Proyección Social en relación a la otras 2 dimensiones (Gestión de la carrera y servicios de apoyo a la formación profesional).

Al respecto, los criterios al respecto señalados por el CONEAU son:

- Proyecto Educativo-Currículo.

- Estrategias de Enseñanza-Aprendizaje.

- Desarrollo de las actividades de Enseñanza -Aprendizaje.

- Evaluación de la Enseñanza -Aprendizaje.

- Estudiantes y Egresados.

- Generación y Evaluación de Proyectos de Investigación.

Los 5 primeros criterios son, principalmente referidos a la enseñanza-aprendizaje.

\section{MODELO DE LAS NORMAS ISO 9000}

Al respecto de las Normas ISO 9000, Farro (2012: 208) sostiene que estas constituyen un modelo, y que "para la implementación de la gestión de procesos académicos y administrativos en la universidad, su valor es incuestionable”. Este es un sistema de aseguramiento de la calidad propiamente, no se expresa como un sistema de gestión de la calidad y proviene del sector industrial. No sirve para acreditar la calidad institucional, sino para partes de ella, como la gestión administrativa y la académica.

Las normas ISO (Farro, 2012) forman un conjunto, siendo la ISO 9000 la referida a los fundamentos, principios y conceptos; el ISO 9001 los requisitos, el ISO 9004 relativo a los planes de mejora y el ISO 19011, que indica la auditoría de la calidad.

En el ISO 9000 - 2000 Farro (2012:213) se proponen 8 requisitos ISO 9000, que son:

1. Objetivos y campo de acción.

2. Normas para consulta.

3. Términos y definiciones.

4. Requisitos del sistema de gestión de calidad.

5. Responsabilidad de la dirección.

6. Gestión de los recursos.

7. Realización del producto y servicio.

8. Medida, análisis y mejora.

En el siguiente gráfico, se muestra la relación de la mejora continua como resultado de la aplicación de un sistema de gestión de la calidad, pero los elementos citados tienen una "traducción" en el Cuadro No 1 (producto, insumo, cliente, procesos como realización del producto) con el agregado de los elementos no directamente operativos: medición, análisis, y mejora, responsabilidad de la Dirección y Gestión de los recursos. 


\section{Gráfico $\mathrm{N}^{\circ} 2$}

Mejora contínua del sistema de gestión de calidad

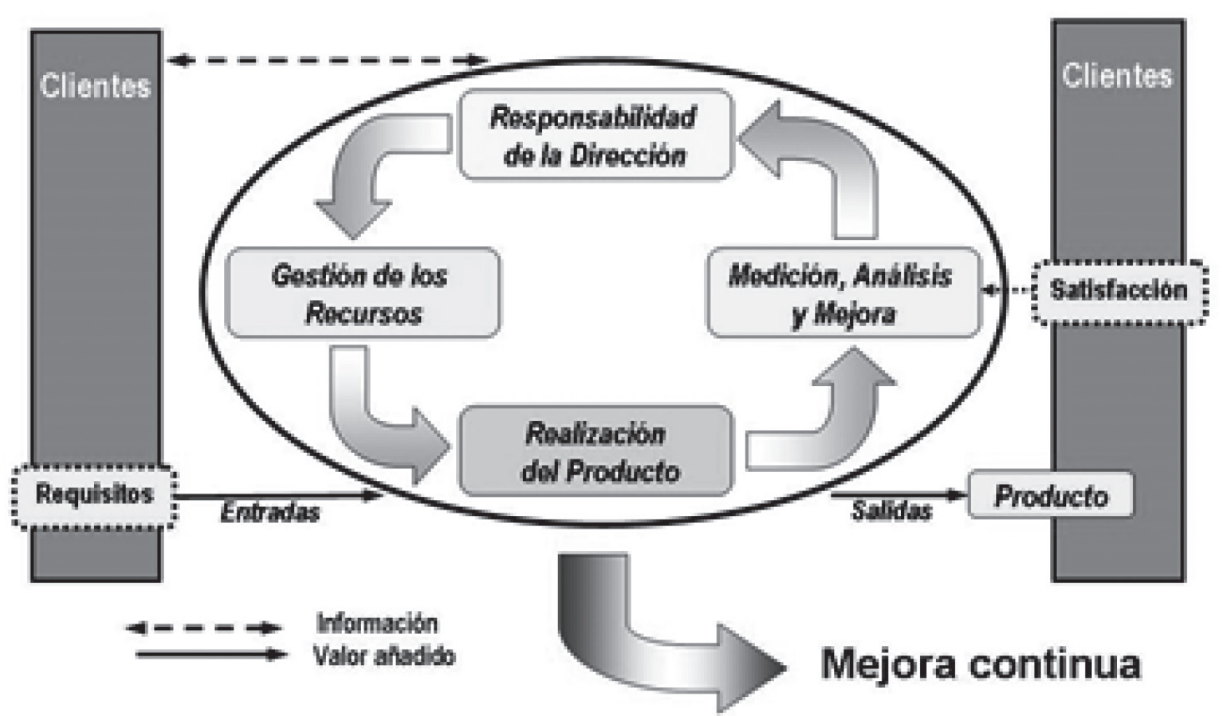

Fuente: http://www.madrid.es/UnidadesDescentralizadas/Cifse/Internet/5CalidadyMedioAmbiente/Calidad/ISO/ISOProceso.jpg

El Modelo ISO 9000 (Farro 2013: 216) presenta los siguientes criterios ISO 9000 relacionados:

- Control del sistema.

- Proceso operativo.

- Actividades de apoyo.

Según la propia ISO (2000; p. VI) la norma "promueve la adopción de un enfoque basado en procesos cuando se desarrolla, implementa y mejora la eficacia de un sistema de gestión de la calidad, para aumentar la satisfacción del cliente mediante el cumplimiento de sus requisitos".

Continúa aclarando que:

"Para que una organización funcione de manera eficaz tiene que indicar y gestionar numerosas actividades relacionadas entre sí. Una actividad que utiliza recursos, y que se gestiona con el fin de permitir que los elementos de entrada se transformen en resultados, se puede considerar como un proceso. Frecuentemente el resultado de un proceso, constituye directamente el elemento de entrada del siguiente proceso".

\section{¿QUÉ DEBE CONTENER UN SISTEMA DE GESTIÓN DE LA CALIDAD?}

Las primeras menciones al Sistema de la Gestión de la Calidad surgen a partir de las normas ISO, y se han extendido, constituyendo ya una doctrina aceptada, basada en los principios ya citados, que asume CONEAU en su modelo de calidad.

¿Cuáles son los requisitos y condiciones de un sistema de gestión de la calidad? ISO 9000 propone los requisitos formales de un sistema de gestión de calidad, los requisitos generales que tienen características de funciones y requisitos documentarios.

En especificidad, el acápite 4 del ISO 9004, de los requisitos, titulado Sistema de Gestión de la Calidad, presenta al respecto:

\subsection{Requisitos generales}

\subsection{Requisitos de la documentación}

4.2.1 Generalidades

4.2.2 Manual de la calidad

4.2.3 Control de los documentos

4.2.4 Control de los registros 
Entre los requisitos generales del sistema de gestión de la calidad en esta norma (ISO, 2000; 2) tenemos:

a) Identificar los procesos necesarios para el sistema de gestión de la calidad y su aplicación a través de la organización,

b) Determinar la secuencia e interacción de estos procesos,

c) Determinar los criterios y métodos necesarios para asegurarse de que tanto la operación como el control de estos procesos sean eficaces,

d) Asegurarse de la disponibilidad de recursos e información necesarios para apoyar la operación y el seguimiento de estos procesos,

e) Realizar el seguimiento, la medición el análisis de estos procesos, $\mathrm{e}$

f) Implementar las acciones necesarias para alcanzar los resultados planificados y la mejora continua de estos procesos.

Al plantear los requisitos de documentación (ISO, 2000; 2-3) estos deben incluir: a) Declaraciones documentadas de una política de calidad y de objetivos de la calidad,

b) Un manual de calidad,

c) Los procedimientos documentados requeridos en esta norma internacional,

d) Los documentos necesitados por la organización para asegurarse de la eficaz planificación, operación $y$ control de sus procesos, $y$

e) Los registros requeridos por esta Norma Internacional.

\section{EL PROCESO CENTRAL EN LAS INSTITU- CIONES DE ENSEÑANZA EN LOS ENFO- QUES DE CONEAU E ISO 9000}

Los modelos de Gestión de la Calidad mencionados (ISO 9000 y otros) no se originan en el ámbito del servicio educativo, sino en el ámbito manufacturero, inicialmente y de servicio, posteriormente, lo cual obliga a hacer la siguiente definición de los conceptos para su aplicación.

\section{Cuadro $\mathrm{N}^{\circ}$ 1:}

$\begin{array}{ll}\text { Producto: } & \text { Formando al cabo del proceso de formación profesional (egresado). } \\ \text { Insumo: } & \text { Formando al inicio del proceso de formación profesional. } \\ \text { Cliente: } & \begin{array}{l}\text { 1. Empleador del formando egresado. } \\ \text { 2. La sociedad, en sus requerimientos de profesionales adecuados a sus necesi- } \\ \text { dades de desarrollo. }\end{array} \\ & \begin{array}{l}\text { 3. El propio formando demanda servicios de formación profesional. } \\ \text { 4. La empresa y la-sociedad demandan resultados de investigación. }\end{array} \\ \text { Proceso: } & \text {-Principal: Enseñanza - Aprendizaje. } \\ \text { Mercado: } & \text {-Secundario: Servicio administrativo. } \\ & \text { Mercado empleador }\end{array}$

Ambos modelos confluyen en el diseño del proceso (producto) y su innovación en relación con las necesidades (requisitos) del mercado. Este diseño e innovación se centra en las características deseadas del producto que son en el caso de una institución educativa, el perfil profesional del egresado conforme a las demandas del mercado ocupacional profesional. Por lo cual, conforme a las relaciones de causalidad presentes en el Modelo de Calidad de CONEAU, se da la siguiente secuencia: 


\section{Gráfico $\mathrm{N}^{\circ} 3$ \\ Mejora contínua del sistema de gestión de calidad}

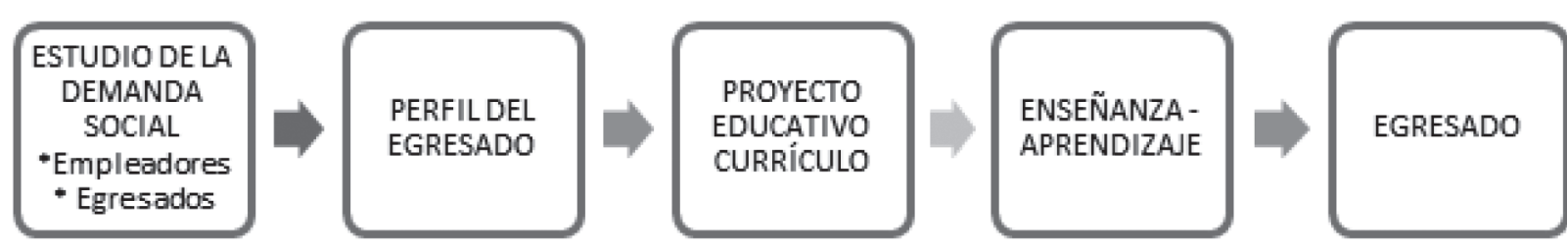

Elaboración: Propia.

Este será el proceso central del sistema de gestión de la calidad, pues el currículo es la planeación del proceso de Enseñanza - Aprendizaje y condiciona las Estrategias de Enseñanza - Aprendizaje, el desarrollo de las actividades de Enseñanza - Aprendizaje y la Evaluación de los Aprendizajes. Últimamente, se refleja en los estudiantes y egresados.

Esta revisión curricular fortalecida con el estudio de la demanda social y mercado ocupacional en forma permanente representa el corazón del proceso de aseguramiento de la calidad y la innovación.

\section{CONCLUSIONES}

Del análisis de los dos modelos indicados, ISO 9000 y CONEAU, se puede indicar lo siguiente:

1. Existe una coherencia entre los mismos, que se origina en el hecho que ellos se influencian por los nuevos enfoques administrativos, con sus rasgos distintivos y vinculados, tales como: enfoque de procesos, calidad total, mejora continua, enfoque sistemático y procesual. Los conceptos de Deming y otros exponentes modernos de la teoría administrativa, están incluidos expresamente en los modelos mencionados, por lo cual es posible el aporte de cada modelo en la formulación de un Sistema de Gestión de la Calidad de la Unidad de Posgrado de la Facultad de Ciencias Contables UNMSM.

2. Los modelos de gestión de la calidad mencionados como el ISO 9000, a diferencia a la de CONEAU, y en especificidad el ISO 9000, se originan inicialmente en el ámbito manufacturero; no en el servicio educativo, al cual se aplica posteriormente, y obliga a realizar la definición adaptativa de los conceptos: producto, insumo, cliente, proceso principal y secundario, y mercado (Ver Cuadro $\mathrm{N}^{\circ} 1$ ).

3. El proceso principal en el caso de las instituciones educativas superiores, "el corazón del negocio" es el de la Enseñanza-Aprendizaje, principalmente, y el de investigación, adicionalmente, los cuales son igualmente priorizados en el modelo de CONEAU, el cual lo considera el proceso básico (enseñanza aprendizaje), y en el que (Dimensión 2: formación profesional) concreta el mayor número de criterios (7 de 16), considerándose también la investigación.

4. Ambos modelos confluyen en el diseño del proceso (producto) y su innovación en relación con las necesidades (requisitos) del mercado. Este diseño e innovación se centra en las características deseadas del producto que es en el presente, perfil profesional del egresado conforme a las demandas del mercado ocupacional profesional. (Ver Gráfico $\mathrm{N}^{\circ} 3$ ).

5. Un sistema de gestión de la calidad debe unir armónica y coherentemente las diversas áreas de actividad de las instituciones de educación superior; actividades que son parte del diseño organizacional de la institución de educación superior $y$ de las innovaciones generadas por los nuevos enfoques pedagógicos y administrativos así como, la cultura de la acreditación. Todo ello, informado y armonizado con una política de calidad que debe hacerse necesariamente explícita. 


\section{REFERENCIAS BIBLIOGRÁFICAS}

1. Consejo de Evaluación, Acreditación y Certificación de la Calidad de la Educación Superior Universitaria - CONEAU - Modelo de Calidad para la acreditación de Programas de Posgrado, modalidad presencia, y estándares ' para Maestrías y Doctorados. Diario Oficial "El Peruano", 23 de diciembre de 2010.

2. ISO. International Standard Organization. 2000. Norma Internacional ISO 9001. Sistemas de gestión de la calidad. Requisitos. Edición ISO. Suiza.

3. FLORES KONJA, Alejandro e HIDALGO, M. (2012) Modelos de Calidad en el marco normativo de la acreditación de las universidades del Perú. Artículo En: Quipukamayoq, Revista de Investigación Contable. Vol. 20 . No 37 - Enero Junio 2012.

4. CONEAU. Consejo de Evaluación, Acreditación y Certificación de la calidad de la Educación Superior Universitaria. Dirección de Evaluación y Acreditación. 2008. Modelo de Calidad para la Acreditación de Carreras Universitarias y Estándares para la Carrera de Educación. Lima 2008. Disponible en: www.oei.es/pdfs/modelo_calidad_acreditacion_universitaria.pdf

5. FARRO CUSTODIO, Francisco. (2012) Planeamiento y gestión estratégica para universidades peruanas competitivas. Ediciones Ugraph. Lima, 2012.

6. Facultad de Ciencias Contables- Universidad Nacional Mayor de San Marcos. Modelo educativo San Marcos - Escuela Académico Profesional de Contabilidad.

7. Facultad de Ciencias Contables- Universidad Nacional Mayor de San Marcos, Plan Estratégico 2012 - 2021. Lima, 2013.

8. Universidad Na cional Mayor de San Marcos. 2012. Plan Estratégico Institucional. 2012 -2021. Lima 2012.

\section{ANEXO No 1 \\ PROPUESTA DE UNA POLÍTICA DE CALIDAD PARA LA UNIDAD DE POSGRADO}

\section{Elementos a considerar por una Política de Calidad}

Muchos elementos considerados en el sistema de gestión de la calidad propuesto por el ISO 9000 y el modelo de CONEAU, en realidad son instrumentos normativos para la Unidad de Posgrado, cuya formulación es mandatoria por disposición interna, dentro del proceso de planificación estratégica de la universidad (UNMSM, 2012). Por ejemplo, en el Manual de Organización y Funciones (MOF) contiene el organigrama: Manual de Procedimientos Administrativos, otros no están previstos como las instrucciones de trabajo. Es decir, la parte correspondiente al "Manual de Procedimientos" ya es conocida y está determinada.

Lo que no se ha considerado es el aspecto del Manual de Calidad, con la determinación en primer lugar de la "Política de Calidad", que es el direccionamiento primordial, lo cual también debe ser enunciada por área.

El esquema que se presenta expresa los elementos que según la aplicación del ISO 9000, integran el Sistema de Gestión de la Calidad, que divide claramente a los mismos en dos grandes agrupaciones: Manual de Calidad y Manual de Procedimientos. 


\section{Gráfico $\mathrm{N}^{\circ} 4$}

Sistema de gestión de la calidad

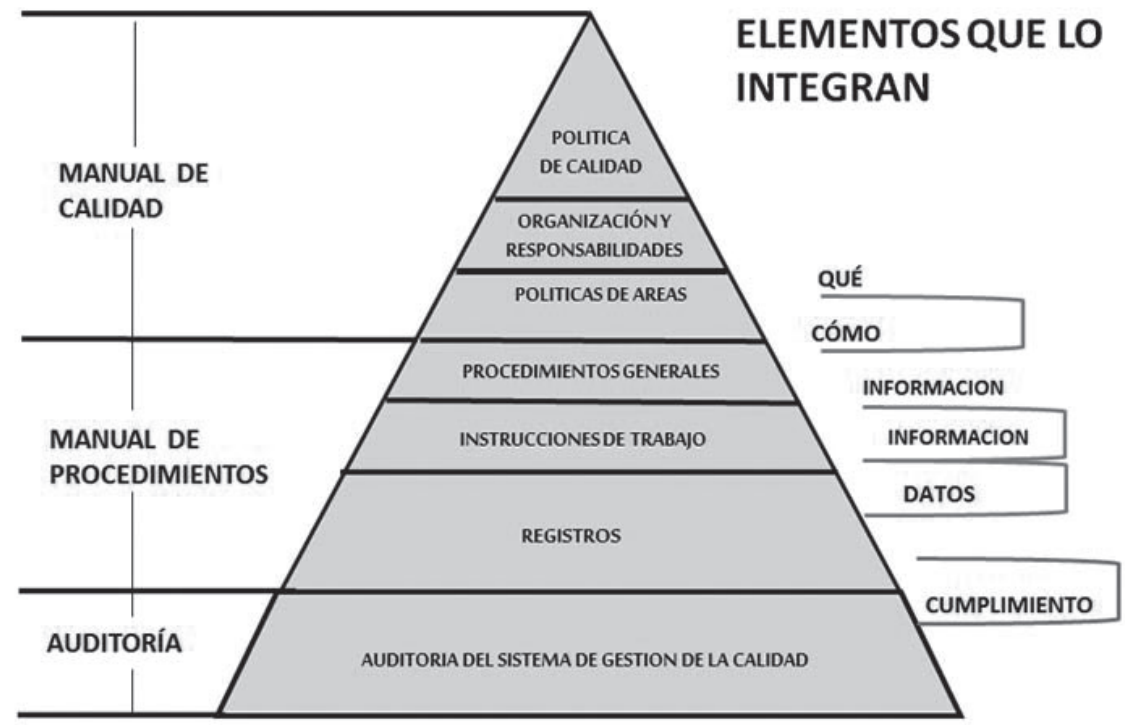

Elaboración: Propia.

Fuente: http://dc347.4shared.com/doc/yN1qv8id/preview_html_m941adae.jpg

Lo que se expresa en el gráfico es que los diferentes sistemas existentes en la unidad - inclusive los que se deben crear con motivos de la autoevaluación y acreditación -- deben mostrar la coherencia de sistemas verdaderamente integrados, para lo cual el enfoque de conjunto es imprescindible y el Sistema de Gestión de la Calidad es vital para asegurar la coherencia, dando a su vez instrumentos operativos, como son: documentos, procedimientos y registros, resultando que el procedimiento de control sea expeditivo.

Según lo expresado, la política de calidad debe dirigirse en primer lugar a la planeación curricular, que conlleva a la siguiente formulación:

\section{PROPUESTA DE POLITÍCAS DE CALIDAD PARA LA UNIDAD DE POSGRADO DE LA FACULTAD DE CIENCIAS CONTABLES}

1. La revisión y actualización curricular se realiza en forma permanente, mínimamente cada tres años, mediante un procedimiento establecido por la Unidad de Posgrado de la Facultad de Ciencias Contables, que determina los plazos temporales y los responsables de la actividad, usando como información primaria básica el estudio de demanda social y mercado ocupacional.

2. Se supervisa el proceso de Enseñanza - Aprendizaje en sus aspectos de: Estrategias didácticas, desarrollo del proceso, evaluación de la Enseñanza Aprendizaje.

3. Se promueve la investigación, principalmente, en relación a la sustentación de las tesis de grado.

4. Se relaciona la investigación formativa con el proceso de Enseñanza - Aprendizaje.

5. Los procesos administrativos principales, de los cuales se tiene mapas de proceso, se rigen por normas y procedimientos de calidad, aprobados por los organismos correspondientes y comunicados. 